\title{
Fast quantum limited read-out of a superconducting qubit using a slow oscillator
}

\author{
Göran Johansson, Lars Tornberg, C.M. Wilson \\ Microtechnology and Nanoscience, MC2, Chalmers, S-412 96 Göteborg, Sweden
}

\begin{abstract}
We describe how to perform fast quantum limited read-out of a solid state qubit biased at its degeneracy point. The method is based on homodyne detection of the phase of a microwave signal reflected by a slow oscillator coupled to the qubit. Analyzing the whole quantum read-out process, we find that the detection is indeed quantum limited and that this limit may be reached even using a resonance circuit with a low quality factor, thus enabling the use of short measurement pulses. As an example, we discuss in detail the read-out of a Cooper-pair box capacitively coupled to a lumped element LC-oscillator. Furthermore, we give formulas for the backaction while not measuring, and discuss optimal parameters for a realistic design capable of fast $(\sim 50 \mathrm{~ns})$ single-shot read-out.
\end{abstract}

Superconducting qubits are strong contenders in the race to build a quantum computer 1 ]. Accordingly, a great deal of effort has gone into developing fast and accurate single-shot read-outs for these devices.

Speed is one important characteristic for a read-out. For accurate qubit detection, the read-out has to be faster than the qubit relaxation time. Moreover, to implement quantum error correction, the read-out must be quicker than the decoherence time.

Another figure of merit, which characterizes the backaction, is the quantum efficiency, $\eta$. According to fundamental principles of quantum measurement, the measurement time, $t_{m s}$, needed to distinguish the two qubit states is always longer or equal to half the dephasing time, $t_{\varphi}$, after which the qubit has lost its quantum coherence 2]. Thus, the quantum efficiency has an upper bound of unity, $\eta=t_{\varphi} / 2 t_{m s} \leq 1$.

Recently, there has been increasing interest in dispersive read-outs for superconducting qubits $[3,4,5,6]$, which measure the reactive response of an oscillator coupled to the qubit. The energy needed for detection is then dissipated far away from the qubit, giving the schemes low backaction. Also, many dispersive read-outs work with the qubit biased at its degeneracy point where the decoherence induced by low frequency fluctuations is minimized [7, 8].

In this letter, we consider how to optimize a dispersive qubit read-out for speed, while maintaining high quantum effieciency and minimizing decoherence. At Yale, Wallraff et al. coupled a charge qubit to a cavity made from a coplanar waveguide $(\mathrm{CPW})[\underline{6}]$. In this experiment, a cavity with a high quality factor $(Q)$ was used to enhance the dispersive phase shift of detected photons. However, using a high- $Q$ cavity severely limits the readout speed, naturally leading us to consider using a low- $Q$ resonator instead. That said, low- $Q$ reduces the dispersive phase shift and implies the need for large detuning between the cavity and qubit, because qubit relaxation is strongly enhanced if the qubit frequency lies within the cavity resonance. Bertet et al. [9] coupled a flux qubit to a low-frequency SQUID oscillator. Unfortunately, they found that the thermal photon noise of the oscillator, present even when not measuring, limited the qubit coherence.

We attempt to harmonize these apparently contradictory requirements. The standard methods of cavity QED [14, 15] are not appropriate for this analysis because they only treat the high- $Q$ limit. Therefore, we take a new approach, based on the quantum network theory introduced by Yurke and Denker [13], which gives analytic results for arbitrary values of $Q$. Indeed, we find that the read-out is quantum limited $(\eta=1)$ independent of $Q$. We go on to calculate the thermal dephasing time in the absence of measurement, $t_{\varphi}^{\text {off }}$. We find that low $Q$ can compensate the effects of thermal photon noise on $t_{\varphi}^{\text {off }}$ by suppressing low-frequency photon fluctuations. We also find that large detuning compensates for a the smaller phase shift by accommodating stronger driving. We go on to discuss optimal parameters for a realistic single-shot read-out.

Our approach is general, and we use it to derive both the Hamiltonians for the measurement of the effective capacitance (inductance) of a charge (flux) qubit. However, we focus the optimization on the Cooper-pair box operated as a charge qubit [10], capacitively coupled to a low-frequency, lumped-circuit LC-resonator. In this setup, the effective capacitance of the Cooper-pair box was recently measured by two different groups [11, 12].

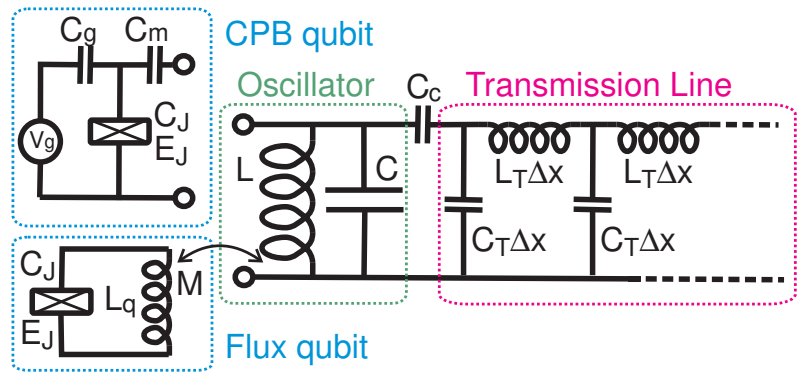

FIG. 1: A CPB (flux) qubit capacitively (inductively) coupled to an LC-oscillator, which is attached to a transmission line.

The Cooper-pair box qubit consists of a small superconducting island coupled to a superconducting reservoir through a Josephson junction characterized by its capacitance, $C_{J}$, and Josephson energy, $E_{J}$. The island is 
coupled to a gate voltage, $V_{g}$, through the gate capacitance, $C_{g}$, and to the LC-oscillator via the coupling capacitance, $C_{m}$. The oscillator is in turn coupled, via $C_{c}$, to a transmission line, here modelled as a semi-infinite line of LC-oscillators. The transmission line is characterized by its capacitance, $C_{T}$, and inductance, $L_{T}$, per unit length. (See Fig. 1)

Since we have a Josephson junction in our circuit, we choose as our coordinates the phase differences across the circuit elements $\phi_{\alpha}(t)=\int^{t} d t^{\prime} V_{\alpha}\left(t^{\prime}\right)$. In the transmission line, we number the phases across the capacitances starting from the oscillator, giving the classical Lagrangian 16 .

$$
\begin{aligned}
L & =\frac{C_{q b} \dot{\phi}_{J}^{2}}{2}-\frac{C_{m}}{2} \dot{\phi}_{C} \dot{\phi}_{J}-C_{g} V_{g} \dot{\phi}_{J}+E_{J} \cos \phi_{J}+ \\
& +\frac{\left(C_{o s c}+C_{c}\right) \dot{\phi}_{C}^{2}}{2}+\frac{C_{c} \dot{\phi}_{1}^{2}}{2}-\frac{C_{c}}{2} \dot{\phi}_{C} \dot{\phi}_{1}-\frac{\phi_{C}^{2}}{2 L}+ \\
& +\sum_{i=1}^{\infty} \Delta x\left(\frac{C_{T} \dot{\phi}_{i}^{2}}{2}-\frac{\left(\phi_{i+1}-\phi_{i}\right)^{2}}{2 L_{T}(\Delta x)^{2}}\right)
\end{aligned}
$$

where $C_{q b}=C_{J}+C_{g}+C_{m}, C_{o s c}=C+C_{m}$. Conjugate to each coordinate $\phi_{\alpha}$, the momentum $q_{\alpha}$ has the dimension of charge, and $\left[\phi_{\alpha}, q_{\beta}\right]=i \hbar \delta_{\alpha \beta}$.

During read-out the oscillator and transmission line charges $\left(q_{C}\right.$ and $\left.q_{1}\right)$ will be driven harmonically at a frequency close to the bare oscillator frequency $\omega_{0}=$ $1 / \sqrt{L\left(C_{o s c}+C_{c}\right)}$, which we consider much lower than the qubit frequency $\omega_{q b}=E_{J} / \hbar$. The amplitude of the charge oscillations induced on the qubit island is determined by the driving strength, which we characterize by a parameter $\beta$ through

$$
\kappa \frac{\left\langle q_{C}(t)+q_{1}(t)\right\rangle_{\max }}{e}=\beta \frac{E_{J}}{4 E_{C}},
$$

where $E_{C}=e^{2} / 2 C_{q b}$ is the charging energy of the qubit and $\kappa=C_{m} / C_{o s c} \ll 1$. For $\beta<1$, we can neglect Landau-Zener transitions and the qubit will follow the oscillator dynamics adiabatically.

At the charge degeneracy point, we rotate the full Hamiltonian to the qubit eigenbasis $\left(\sigma_{x} \leftrightarrow \sigma_{z}\right)$ and expand the eigenenergies to second order in the induced charge fluctuations

$$
\begin{aligned}
H & =-\frac{E_{J}}{2} \sigma_{z}+\left(\frac{1}{2 C_{o s c}}-\frac{g_{C}}{2} \sigma_{z}\right)\left(q_{C}+q_{1}\right)^{2}+\frac{\phi_{C}^{2}}{2 L}+ \\
& +\frac{q_{1}^{2}}{2 C_{c}}+\frac{1}{\Delta x} \sum_{i=1}^{\infty}\left(\frac{\left(q_{i+1}\right)^{2}}{2 C_{T}}+\frac{\left(\phi_{i+1}-\phi_{i}\right)^{2}}{2 L_{T}}\right)
\end{aligned}
$$

where $g_{C}=8 \kappa^{2} E_{C}^{2} / e^{2} E_{J}$ indicates the qubit-oscillator coupling. From the qubit perspective, the slow transverse charge fluctuations induced by the oscillator result in second order longitudinal fluctuations as described by the $\left(q_{C}+q_{1}\right)^{2} \sigma_{z}$-term $[17$. The same term causes a state dependent shift in the oscillator's electrostatic energy. This may be modelled as an effective oscillator capacitance $C_{o s c}^{g / e}=C_{o s c} \pm g_{C} C_{o s c}^{2} \equiv C_{o s c} \pm C_{Q}$, where the last term is the state dependent quantum capacitance $C_{Q}$. This in turn gives different oscillator resonance frequencies for the qubit in the ground/excited state $\omega_{0}^{g / e}=\omega_{0}\left(1 \mp C_{Q} / 2\left(C_{o s c}+C_{c}\right)\right)$, which was recently experimentally measured in Refs. 11, 12].

The same type of analysis can be done for the flux qubit in Fig. [16]. The oscillator part of this Hamiltonian is

$$
H_{\text {flux }}^{o s c}=\frac{q_{C}^{2}}{2 C}+\left(\frac{1}{2 L}-\frac{g_{L}}{2} \sigma_{z}\right) \phi_{C}^{2}
$$

where now the qubit-oscillator coupling is $g_{L}=$ $2 M^{2}\left\langle\phi_{J}\right\rangle^{2} / \Delta L_{q}^{2} L^{2}$. Here $\left\langle\phi_{J}\right\rangle$ is the average flux generated by the circulating current in the Josephson loop, $M$ is the mutual inductance between the oscillator and qubit, $L_{q}$ is the qubit inductance and $\Delta$ is the qubit level splitting. In this environment, the Josephson junction acts as an effective inductance $L_{o s c}^{g / e}=L \pm L^{2} g_{L}$ giving the state dependent resonance frequencies $\omega_{0}^{g / e}=$ $\omega_{0}\left(1 \mp L g_{L} / 2\right)$.

The equations for the transmission line coordinates and momenta, in the limit $\Delta x \rightarrow 0$, correspond to the massless Klein-Gordon equation, which has traveling wave solutions, $\phi(x, t)=\phi_{\text {in }}(t-x / v)+\phi_{\text {out }}(t+x / v)$, with components moving towards and away from the oscillator with a velocity $v=1 / \sqrt{L_{T} C_{T}}$. The driving source determines the in-field, $\phi_{i n}(t-x / v)$, from which we may derive the detectable out-field, $\phi_{\text {out }}(t+x / v)$, as well as the charge field $q(t)=q_{C}(t)+q_{1}(t)$ and phase field $\phi_{C}(t)$, giving the backaction on the charge and flux qubit, respectively. In so doing, we solve the Heisenberg equations of motion corresponding to the Hamiltonian in Eq. (3). Since they are linear, this is straightforward in the Fourier representation. The linearity is a consequence of the weak coupling to the qubit, which is the only nonlinear circuit element. However, we are free to consider any strength of the oscillator-transmission line-coupling.

First analyzing the charge qubit read-out we define the state-dependent transconductance $\chi^{g / e}(\omega)=$ $q^{g / e}(\omega) / \phi_{i n}(\omega)$, and write the general solution

$$
\begin{aligned}
& \phi_{\text {out }}^{g / e}(\omega)=\frac{\chi^{g / e}(\omega)}{\left(\chi^{g / e}(\omega)\right)^{*}} \phi_{\text {in }}(\omega)=e^{i \varphi_{r}^{g / e}(\omega)} \phi_{\text {in }}(\omega), \\
& \chi^{g / e}(\omega)=\frac{i 2 C_{c} C_{\text {osc }}^{g / e} L \omega^{3}}{1-\left(C_{\text {osc }}^{g / e}+C_{c}\right) L \omega^{2}-i \omega C_{c} Z_{0}\left(1-L \omega^{2} C_{\text {osc }}^{g / e}\right)},
\end{aligned}
$$

where $Z_{0}=\sqrt{L_{T} / C_{T}}$ is the characteristic impedance of the transmission line. Since there is no dissipation in the lumped circuit, we have $\left|\phi_{g / e}^{\text {out }}(\omega)\right|=\left|\phi^{\text {in }}(\omega)\right|$, and the reflected signal is characterized by a frequency dependent phase shift $\varphi_{r}^{g / e}(\omega)$. By introducing creation and annihilation operators for the in-field satisfying the commutation relations $\left[a_{\omega}, a_{\omega^{\prime}}^{\dagger}\right]=\delta\left(\omega-\omega^{\prime}\right)$ and $\left[a_{\omega}, a_{\omega^{\prime}}\right]=0$, 
we can write

$$
\begin{aligned}
\phi_{\text {in }}(x, t) & =N \int_{0}^{\infty} \frac{d \omega}{\sqrt{\omega}}\left(a_{\omega} e^{-i \omega(t-x / v)}+\text { h.c. }\right), \\
\phi_{\text {out }}^{g / e}(x, t) & =N \int_{0}^{\infty} \frac{d \omega}{\sqrt{\omega}}\left(a_{\omega} e^{i\left[\varphi_{r}^{g / e}(\omega)-\omega(t-x / v)\right]}+\text { h.c. }\right), \\
q^{g / e}(t) & =N \int_{0}^{\infty} \frac{d \omega}{\sqrt{\omega}}\left(\chi^{g / e}(\omega) a_{\omega} e^{-i \omega t}+h . c .\right),
\end{aligned}
$$

with normalization $N=\sqrt{\hbar Z_{0} / 4 \pi}$. We model a signal generator by setting the in-field to a coherent state with a narrow-band distribution around the drive frequency $\omega_{d}$ and an intensity corresponding to $\Gamma_{i n}$ photons per second. Using the standard model of homodyne detection, the time $t_{m s}$ needed to distinguish the phase difference $\delta \varphi_{r}=\varphi_{r}^{e}\left(\omega_{d}\right)-\varphi_{r}^{g}\left(\omega_{d}\right)$ in the out-field is $t_{m s}^{-1}=4 \Gamma_{i n} \sin ^{2}\left(\delta \varphi_{r} / 2\right)$ [15]. We can then compare this to the measurement-induced qubit dephasing. In the weak qubit-oscillator coupling regime, we straightforwardly calculate the decay of the off-diagonal element of the reduced qubit density matrix $\left|\left\langle\sigma^{+}(t)\right\rangle\right|=$ $\left|\left\langle\sigma^{+}(0)\right\rangle\right| e^{-t / t_{\varphi}}$ and find the dephasing rate

$$
t_{\varphi}^{-1}=\Gamma_{i n} \frac{\left(g_{C} Z_{0}\right)^{2}}{8 \omega_{d}^{2}}\left(\left|\chi^{g}\left(\omega_{d}\right)\right|^{2}+\left|\chi^{e}\left(\omega_{d}\right)\right|^{2}\right)^{2} .
$$

The quantum efficiency $\eta$ can now be evaluated for arbitrary circuit parameters. First we note that the drive strength $\Gamma_{i n}$ cancels in the expression for $\eta$. The LCoscillator has a quality factor $Q=\left(C_{o s c}+C_{c}\right) / C_{c}^{2} Z_{0} \omega_{0}$, and close to resonance we can approximate Eq. (5) as

$$
\chi^{g / e}(\omega)=-2 \frac{C_{o s c}}{C_{c} Z_{0}}\left[1+i 2 Q\left(\omega-\omega_{0}^{g / e}\right) / \omega_{0}\right]^{-1},
$$

Driving the circuit at the average resonance frequency $\omega_{d}=\left(\omega_{0}^{g}+\omega_{0}^{e}\right) / 2$ the quantum efficiency is

$$
\eta=\frac{t_{\varphi}}{2 t_{m s}}=\frac{\left(x^{-1}+x\right)^{2}}{8} 2 \sin ^{2}[2 \arctan x]=1,
$$

where $x=Q C_{Q} /\left(C_{o s c}+C_{c}\right)$. Somewhat surprisingly, we find a quantum efficiency of unity independent of $Q$ and the coupling to the qubit $C_{Q} / C_{o s c}$. For non-optimal drive frequencies the efficiency is below one. The inefficiency is related to the information stored in the state-dependent delay time of the measurement pulse.

For flux qubit read-out Eq. (7) apply with the substitution $g_{C} \rightarrow g_{L}$ and using the relevant transfer function $\chi(\omega)^{g / e}=\phi_{C}^{g / e}(\omega) / \phi^{i n}(\omega)$. In this case we also find full quantum efficiency independent of $\mathrm{Q}$.

We can now relate this result to the quantum efficiency of some other read-out methods. Detecting the phase of the signal transmitted through the CPW cavity in the Yale experiment has half the optimal efficiency $\eta=1 / 2[14]$, where the inefficiency is related to the reflected signal, which is not detected. In our system, the efficiency is optimal since the whole signal is reflected and detected. In the point contact detector, electrons in a range of energies, as specified by the driving voltage, are used to probe the contact. Thus a particular energy-dependence of the point contact transmission is needed for the efficiency to approach unity [18]. In our system, the signal source is essentially monochromatic, probing the system at a single energy. Thus, there are no constraints on the energy dependence of the scattering, allowing, e.g. for a low- $Q$ oscillator.

Finding full quantum efficiency for a wide range of parameters leads us to consider optimizing the circuit parameters for other criteria. One important figure of merit is the rate of qubit dephasing induced by the measurement device when not measuring. In this case, we assume a thermal distribution with temperature $T$ of the in-field and find the dephasing time

$$
\left(t_{\varphi}^{\mathrm{off}}\right)^{-1}=n\left(\omega_{0}\right)\left[1+n\left(\omega_{0}\right)\right] Q \frac{C_{Q}^{2}}{C_{o s c}^{2}} \omega_{0}
$$

where $n\left(\omega_{0}\right)=1 /\left(e^{\hbar \omega_{0} / k_{B} T}-1\right)$ is the thermal photon occupation number at the oscillator frequency. This expression is similar to what is found for a superconducting flux qubit coupled to a DC SQUID [9]. To minimize offstate dephasing one should cool the oscillator, but it is also advantageous to reduce the oscillator-qubit coupling and to use a low- $Q$ oscillator. Basically, the integral of the spectral density of the fluctuations, the variance, is fixed by thermodynamics, independent of $Q$. Therefore, lowering $Q$ stretches the same power over a wider bandwidth, reducing the low-frequency power.

Another figure of merit is the relaxation time of the qubit, $t_{1}$, induced by the measurement device. We evaluate $t_{1}$ using standard weak coupling expressions [1], giving that it is proportional to the real part of the impedance seen from the qubit, at the qubit frequency $\omega_{q b}$. For the simple oscillator circuit shown in Fig. [1 with a low $Q, t_{1}$ could be as short as a few hundred nanoseconds. The solution to this apparent problem is to add a nondissipative low-pass filter between the transmission line and the oscillator. Due to the large frequency difference between the oscillator and qubit, this is straightforward. In principle, commercially available pi-filter can improve $t_{1}$ by a factor of 1000 while not affecting the low frequency measurement properties. This is well beyond the point where other sources of relaxation will dominate.

Along with these intrinsic figures of merit, we should also consider the performance of the read-out constrained by existing technology. In particular, we want to evaluate the measurement time given that we first amplify the reflected signal with an amplifier characterized by an impedance, $Z_{0}$, and a noise temperature, $T_{N}$, which exceeds the quantum limit. We also assume the input of the amplifier is coupled to a matched load, e.g. using an isolator. Now, the maximum number of photons in 


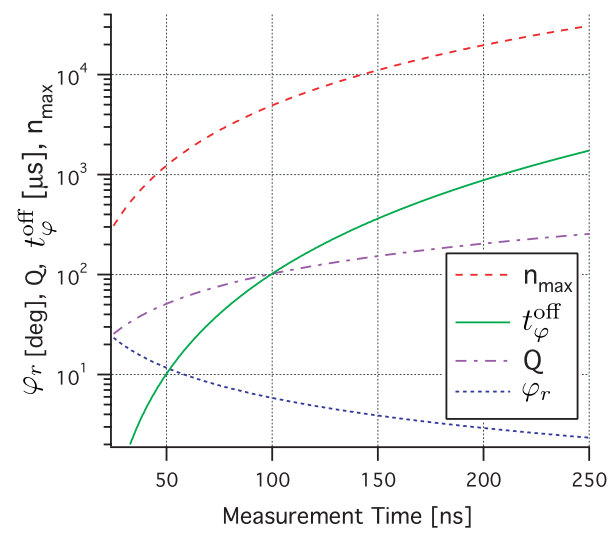

FIG. 2: The number of oscillator photons $\left(n_{\max }\right)$, off-state thermal dephasing time $\left(t_{\phi}^{\text {off }}\right)$, oscillator quality factor $(Q)$, and phase shift $\left(\varphi_{r}\right)$ as a function of read-out time.

the oscillator, $n_{\max }=C_{o s c}\left(e \beta E_{J} / 2 E_{C} C_{m}\right)^{2} / \hbar \omega_{d}$, is determined by how hard we can drive the qubit, which is limited by the width of the quantum capacitance peak in gate charge. Roughly, we can use the half width at half maximum (HWHM) of the peak, giving $\beta=\sqrt{2^{2 / 3}-1} / 2$ in Eq. (2). Taking the phase shift between the ground and excited state as $\varphi_{r}=\arctan \left(4 Q C_{Q} / C_{o s c}\right)$, we can estimate the measurement time as

$$
t_{m s}=8 \frac{n_{a m p}}{n_{\max } \varphi_{r}^{2}} \frac{Q}{\omega_{d}} \approx \frac{Z_{0} k_{B} T_{N} C_{c}^{2}}{4 \beta^{2} \lambda^{2} e^{2}}
$$

where $n_{a m p}=k_{B} T_{N} / \hbar \omega_{d}$ is the number of noise photons in the amplifier, $\lambda=C_{m} / C_{q b}$, and we have assumed a signal-to-noise ratio in phase of 2 , which corresponds to the same definition used for the quantum limit. In the last approximation, we assume $\varphi_{r} \approx 4 Q C_{Q} / C_{o s c}$.

We then want to find a circuit design that gives the fastest measurement time while still protecting the qubit from thermal dephasing. In addition, we require that the measurement time be longer than the response time of the resonant circuit, $\tau_{Q}=Q / \omega_{0}$. This ensures that the reflected measurement pulse is not distorted and that its delay is not state dependent. In Fig. 2, we plot optimized values for $n_{\max }, \varphi_{r}, Q$, and $t_{\phi}^{\text {off }}$ as a function of $t_{m s}$. We assume $t_{m s} / \tau_{Q}=4, T=20 \mathrm{mK}, \lambda=0.5$, $\omega_{0} / 2 \pi=650 \mathrm{MHz}, \omega_{q b} / 2 \pi=5 \mathrm{GHz}, Z_{0}=50 \Omega$ and $T_{N}=0.8 \mathrm{~K}[19]$ which implies $n_{a m p} \approx 25$. We see that measurement times of order $50 \mathrm{~ns}$ are readily achievable while still maintaining off-state dephasing times greater than $10 \mu \mathrm{s}$.

We can compare this result with the estimates of [14], trying to understand how the estimates here are 2-3 orders of magnitude faster. Fundamentally, the speed-up comes from having a much lower $Q$, which determines the response time of the resonator. However, lowering $Q$ alone also decreases $\varphi_{r}$. In fact, if nothing is done to compensate this, the measurement time actually increases as $1 / Q$. The way to compensate is to increase $n_{\max }$. This is where the large detuning of the our proposal is key. In the near-resonant design of [14], $n_{\max }$ is limited by the fact that the shift of the qubit frequency due to photon occupation must be less than the detuning. If this condition is violated, qubit relaxation is strongly enhanced by the cavity. This implies $n_{\max } \approx 100$ and $n_{a m p} / n_{\max } \varphi_{r}^{2} \approx 1$. This is not a limitation for a lowfrequency oscillator, however, allowing for much harder driving. For the the case of $t_{m s}=50 \mathrm{~ns}$ above, we have in fact $n_{o s c} \approx 1200$, maintaining $n_{a m p} / n_{\max } \varphi_{r}^{2} \approx 1 / 2$ despite the small phase shift. In fact, this ratio is constant for the whole range of plotted parameters.

In summary, we have discussed how to optimize a general dispersive qubit read-out for speed, while maintaining maximum quantum efficiency and long dephasing times. We have found that reading out a charge qubit by measuring its quantum capacitance, through the frequency shift of a lumped circuit LC-oscillator, is quantum efficient $(\eta=1)$ independent of the oscillator quality factor. We also find that a low $Q$ protects the qubit from thermal dephasing in the off-state, while increasing the speed of the read-out. Furthermore, we have discussed how to optimize the circuit parameters to achieve singleshot read-out using a commercial amplifier.

We thank Per Delsing, Tim Duty, Vitaly Shumeiko and Margareta Wallquist for useful discussions. This work was supported by the Swedish SSF and VR, by the Wallenberg foundation, and by the EU under the IST-SQUBIT-2 and RSFQUBIT programmes.

[1] G. Wendin and V.S. Shumeiko, in Handbook of Theor. and Comp. Nanotech., eds. M. Rieth and W. Schommers, American Scientific Publishers (2006).

[2] V. Braginski and F. Khalili, Quantum Measurements, Cambridge University Press, (1992).

[3] A. Lupascu et al., Phys. Rev. Lett. 93, 177006 (2004)

[4] M. Grajcar et al., Phys. Rev. B 69, 060501(R) (2004)

[5] I. Siddiqi et al., Phys. Rev. B 73, 054510 (2006)

[6] A. Wallraff et al., Phys. Rev. Lett. 95, 060501 (2005)

[7] O. Astafiev et al., Phys. Rev. Lett. 93, 267007 (2004).

[8] G. Ithier et al., Phys. Rev. B 72, 134519 (2005)

[9] P. Bertet et al., Phys. Rev. Lett. 95, 257002 (2005)

[10] M. Büttiker, Phys. Rev. B 36, 3548 (1987); V. Bouchiat et al., Phys. Scr. T76, 165 (1998); A. Shnirman, G. Schön, and Z. Hermon, Phys. Rev. Lett. 79, 2371 (1997)

[11] M. A. Sillanpää et al., Phys. Rev. Lett.95, 206806 (2005)

[12] T. Duty et al., Phys. Rev. Lett.95, 206807 (2005)

[13] B. Yurke and J. S. Denker Phys. Rev. A 29, 1419 (1984)

[14] A. Blais et al., Phys. Rev. A 69, 062320 (2004)

[15] C. W. Gardiner, P. Zoller, Quantum Noise (SpringerVerlag Berlin Heidelberg, 1991 ,2000)

[16] G. Johansson et al., Condens. Matter 18, S901-S920 (2006).

[17] Y. Makhlin and A. Shnirman, Phys. Rev. Lett. 92, 178301 (2004)

[18] S. Pilgram and M. Büttiker, Phys. Rev. Lett. 89, 200401 
(2002); D. V. Averin and E. V. Sukhorukov Phys. Rev. Lett. 95, 126803 (2005)
[19] Quinstar U-650-2 measured at 4.2K in our laboratory. 